\title{
Non-Boltzmann stationary distributions and nonequilibrium relations in active baths
}

\author{
Aykut Argun, ${ }^{1,2}$ Ali-Reza Moradi, ${ }^{2,3,4}$ Erçağ Pinçe, ${ }^{2}$ Gokhan Baris Bagci,${ }^{5}$ Alberto Imparato, ${ }^{6}$ and Giovanni Volpe ${ }^{1,2,7}$ \\ ${ }^{1}$ Department of Physics, University of Gothenburg, SE-41296 Gothenburg, Sweden \\ ${ }^{2}$ Soft Matter Lab, Department of Physics, Bilkent University, Cankaya, 06800 Ankara, Turkey \\ ${ }^{3}$ Department of Physics, University of Zanjan, P.O. Box 45195-313, Zanjan, Iran \\ ${ }^{4}$ Optics Research Center, Institute for Advanced Studies in Basic Sciences, P.O. Box 45137-66731, Zanjan, Iran \\ ${ }^{5}$ Department of Materials Science and Nanotechnology Engineering, TOBB University of Economics and Technology, 06560 Ankara, Turkey \\ ${ }^{6}$ Department of Physics and Astronomy, University of Aarhus Ny Munkegade, Building 1520, DK-8000 Aarhus C, Denmark \\ ${ }^{7}$ UNAM - National Nanotechnology Research Center, Bilkent University, Ankara 06800, Turkey
}

(Received 19 June 2016; published 29 December 2016)

\begin{abstract}
Most natural and engineered processes, such as biomolecular reactions, protein folding, and population dynamics, occur far from equilibrium and therefore cannot be treated within the framework of classical equilibrium thermodynamics. Here we experimentally study how some fundamental thermodynamic quantities and relations are affected by the presence of the nonequilibrium fluctuations associated with an active bath. We show in particular that, as the confinement of the particle increases, the stationary probability distribution of a Brownian particle confined within a harmonic potential becomes non-Boltzmann, featuring a transition from a Gaussian distribution to a heavy-tailed distribution. Because of this, nonequilibrium relations (e.g., the Jarzynski equality and Crooks fluctuation theorem) cannot be applied. We show that these relations can be restored by using the effective potential associated with the stationary probability distribution. We corroborate our experimental findings with theoretical arguments.
\end{abstract}

DOI: 10.1103/PhysRevE.94.062150

\section{INTRODUCTION}

Already at the beginning of the 20th century, classical thermodynamics provided a comprehensive understanding of the equilibrium behavior of macroscopic systems in contact with thermal reservoirs and deterministic relations between their thermodynamic quantities [1]. Instead, it has only been during the past few decades that the thermodynamics of nonequilibrium systems and active matter has become the subject of intense research [2-4]. This interest is motivated by the need to explore the physics of microscopic systems, where thermal fluctuations play a crucial role and introduce stochasticity [5,6], and of living matter, which is intrinsically far from equilibrium $[7,8]$. On the one hand, for microscopic systems thermal fluctuations become essential because of the limited number of degrees of freedom and can generate outcomes that are forbidden in macroscopic systems. For example, the presence of thermal fluctuations allows the violation of the second law for single realizations of a process, even though the second law still holds on average $[5,6,9,10]$. A major advance in stochastic thermodynamics has been the realization that, instead of just averaging the fluctuations out, it is possible to make use of the information they contain in order to recover equilibrium information even from nonequilibrium measurements using, e.g., the Jarzynski equality and the Crooks fluctuation theorem $[2,3,11,12,12-14]$. These relations have recently been tested and verified experimentally for various systems that are coupled to thermal baths [15-24]. On the other hand, many systems, such as living matter, are intrinsically far from equilibrium. For example, biomolecules within the cell are coupled with an active bath due to the presence of molecular motors within the cytoplasm, which leads to striking and largely not yet understood phenomena such as the emergence of anomalous diffusion [7]. Also, protein folding might be facilitated by the presence of active fluctuations [25] and active matter dynamics could play a cen- tral role in several biological functions [26-28]. Until now, the experimental study of all these systems has been limited [8].

Here we present a series of experiments with an optically trapped Brownian particle coupled to an active bath. First, we show that in an active bath the statistical properties of a particle held in a potential do not generally follow the Boltzmann statistics because of the correlated noise introduced by the active bath: As the characteristic scale of the optical trap becomes comparable to the correlation length introduced by the active noise, we observe a transition between a regime that can be described with Boltzmann statistics (albeit at a higher effective temperature) to a regime that follows non-Boltzmann statistics. Then we also show that a major consequence of this fact is that nonequilibrium relations, such as the Crooks fluctuation theorem [14], the Jarzynski equality [11], and the integral fluctuation theorem [12], cannot be applied in active baths according to their classical formulation; We show nevertheless that they can be recovered by introducing an effective potential. We remark that, although breakdown of equilibrium conditions in active systems is not unexpected, the experimental observation of such violations is of great practical interest, because their quantitative and qualitative investigation is crucial to develop better models for active, farfrom-equilibrium, and living systems $[8,29,30]$. Our findings pose some significant limitations to the possibility of applying nonequilibrium fluctuation relations to active matter systems, including living matter, and point to the need for alternative approaches that explicitly model the presence of nonthermal fluctuations, such as the use of effective potentials that play the same role as thermodynamic potentials in passive baths.

\section{NON-BOLTZMANN STATIONARY DISTRIBUTION IN ACTIVE BATHS}

The motion of a Brownian particle immersed in a liquid at temperature $T$ is described by the overdamped 
Langevin equation

$$
\frac{d x}{d t}=-\frac{1}{\gamma} \frac{d V(x)}{d x} d t+\eta_{\mathrm{p}},
$$

where $x$ is the particle position, $\gamma$ is the particle friction coefficient, $V(x)$ is the potential, $\eta_{\mathrm{p}}$ represents the (white) thermal noise characterized by $\left\langle\eta_{\mathrm{p}}(t+\tau) \eta_{\mathrm{p}}(t)\right\rangle=2 D_{\mathrm{p}} \delta(\tau)$, and $D_{\mathrm{p}}$ is the translational diffusion coefficient, which importantly is related to $\gamma$ by the fluctuation-dissipation relation $D_{\mathrm{p}} \gamma=k_{\mathrm{B}} T$. The corresponding position (equilibrium) distribution follows a Boltzmann distribution

$$
p(x)=Z^{-1} \exp \left(-\frac{V(x)}{k_{\mathrm{B}} T}\right),
$$

where $Z=\int_{-\infty}^{+\infty} \exp [-\beta V(x)] d x$ is the partition function.

Experimentally we employ a spherical Brownian microparticle (polystyrene, diameter $2 R=4.06 \pm 0.20 \mu \mathrm{m}$ ) in a watery solution trapped by an optical trap generated by focusing a laser beam (wavelength $\lambda=976 \mathrm{~nm}$ ) with a highnumerical-aperture objective [oil immersion, $100 \times$, numerical aperture (NA) equal to 1.30] [31]. The optical potential is harmonic:

$$
V_{\mathrm{ot}}(x)=\frac{1}{2} k x^{2}
$$

with stiffness $k$, which is proportional to the laser intensity and can therefore be adjusted by using some neutral density filters along the laser beam path. The expected Boltzmann position distribution (2) is Gaussian:

$$
p_{\mathrm{ot}}(x)=Z_{\mathrm{ot}}^{-1} \exp \left(-\frac{k x^{2}}{2 k_{\mathrm{B}} T}\right),
$$

where $Z_{\mathrm{ot}}=\sqrt{\frac{2 \pi k_{\mathrm{B}} T}{k}}$ is the normalization prefactor. The particle position is monitored by digital video microscopy (using the radial symmetry algorithm [32]) with a spatial resolution of $5 \mathrm{~nm}$. The results are plotted by the blue symbols in Figs. 1(a), 1(b), and 1(c) for $k=0.42,3.6$, and $22 \mathrm{fN} \mathrm{nm}^{-1}$, respectively. As expected from Eq. (4), the resulting distributions are Gaussian with variance proportional to $k^{-1}$ [dashed blue lines in Figs. 1(a)-1(c)] and are in very good agreement with the experimental data. The characteristic time scale with which the particle is attracted towards the center of the harmonic trapping potential is given by $\tau_{\mathrm{ot}}=$
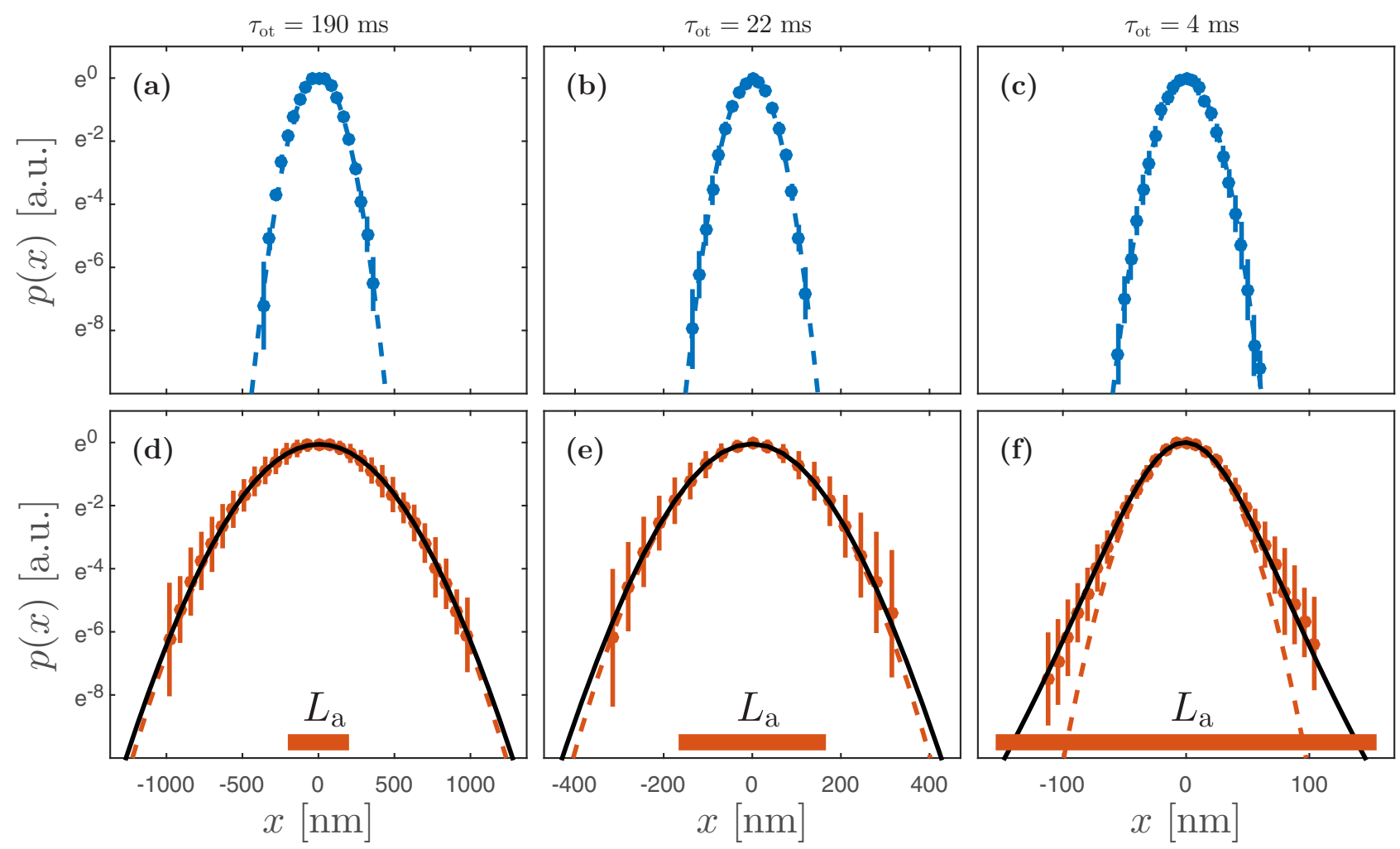

FIG. 1. Emergence of non-Boltzmann position distributions in active baths. Measured position distributions (blue symbols) and corresponding Gaussian distributions (dashed blue lines) are shown for a Brownian particle in a thermal bath confined in a harmonic trapping potential with trapping stiffness (a) $k=0.42 \mathrm{fN} \mathrm{nm}^{-1}$, (b) $k=3.6 \mathrm{fN} \mathrm{nm}^{-1}$, and (c) $k=22 \mathrm{fN} \mathrm{nm}^{-1}$. As $k$ is increased, the particle becomes more confined, but the distribution remains Gaussian. Note the different scales for the position axes. Also shown are measured position distributions (red symbols) and best Gaussian fits (red dashed lines) for a Brownian particle in an active bath in a harmonic trapping potential with trapping stiffness (d) $k=0.42 \mathrm{fN} \mathrm{nm}^{-1}$, (e) $k=3.6 \mathrm{fN} \mathrm{nm}^{-1}$, and (f) $k=22 \mathrm{fN} \mathrm{nm}^{-1}$. As $k$ is increased, the particle gets confined within a length scale comparable to the persistence length in the active bath $\left[L_{\mathrm{a}}\right.$, red bars in (d)-(f)]; consequently, the distribution deviates from Gaussian and can be fitted with a heavy-tailed $q$-Gaussian distribution (solid black line) with (d) $q=1.013$, (e) $q=1.023$, and (f) $q=1.142$ [(a) $q=0.997$, (b) 1.000 , and (c) 1.001 , compatibly with a Gaussian profile]. The data are obtained from 200-s-long trajectories acquired at about 400 frames per second (fps); the error bars are obtained by repeating the measurements six times. 

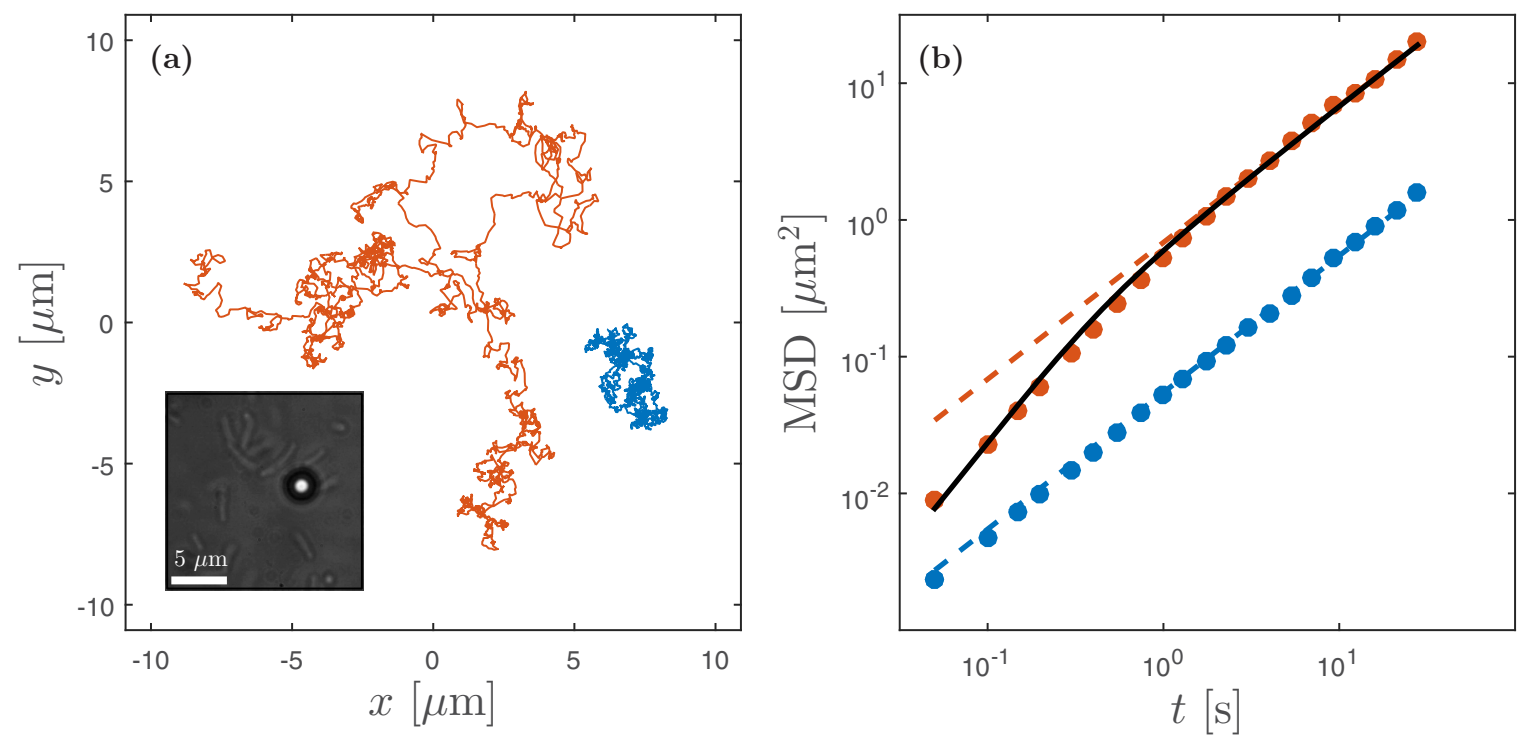

FIG. 2. Particle in an active bath. (a) Sample trajectories of a microscopic particle (polystyrene, diameter $2 R=4.06 \pm 0.20 \mu \mathrm{m}$ ) in an active (bacterial) bath (red line) and in a thermal bath (blue line). The trajectories are extracted from a 150 -s video sampled at 20 fps. The inset shows a sample frame from the acquired video in the active bath; the bright spot corresponds to the particle, while the elongated lighter objects are the bacteria. (b) The MSD of the particle in the active bath along one axis (red symbols) features a transition from a superdiffusive regime at short time scales to enhanced diffusion at long time scales, differently from the MSD of the particle in the thermal bath (blue symbols).

$\gamma / k$ [31]; we obtain $\tau_{\mathrm{ot}}=190,22$, and $4 \mathrm{~ms}$ for the data reported in Figs. 1(a), 1(b), and 1(c), respectively.

We now consider a Brownian particle in an active bath. We realize the active bath by adding motile bacteria to the watery solution where the particle is immersed [33,34] [inset in Fig. 2(a)]: The bacteria behave as active particles and exert nonthermal forces on our probe particle so that it experiences nonthermal fluctuations and features a qualitatively different behavior from that of a Brownian particle in a thermal bath. We do not observe any accumulation of bacteria around the focal spot due to optical forces; this can be understood because the optical forces acting on the bacteria (which have a small polarizability due to their small size and low refractive index mismatch) are much weaker than self-propulsion forces. The Langevin equation that describes this system is

$$
\frac{d x}{d t}=-\frac{1}{\gamma} \frac{d V(x)}{d x} d t+\eta_{\mathrm{p}}+\eta_{\mathrm{a}}
$$

where $\eta_{\mathrm{a}}$ represents the fluctuations due to the presence of the active bath. This is an additional source of noise that must be added to Eq. (1) in order to describe the dynamics of the particle; unlike thermal fluctuations, these fluctuations are exponentially correlated over time so that $\left\langle\eta_{\mathrm{a}}(t+\tau) \eta_{\mathrm{a}}(t)\right\rangle=$ $\frac{L_{\mathrm{a}}^{2}}{\tau_{\mathrm{a}}^{2}} \exp \left(-\tau / \tau_{\mathrm{a}}\right)$, where $\tau_{\mathrm{a}}$ is the persistence time of the bacterial forces acting on the particle associated with a persistence length $L_{\mathrm{a}}$, i.e., a characteristic distance along which the bacteria drag (on average) the particle [33-36]. It is important to remark that, even though the correlation is the same as in an Ornstein-Uhlenbeck process, the noise $\eta_{\mathrm{a}}$ appearing in Eq. (5) is not Gaussian and cannot be modeled with OrnsteinUhlenbeck noise [8] because it arises as a consequence of the swimming bacteria in the active bath, which self-propel with constant forces that randomly change direction [37]. Importantly, unlike the outcome in the case of Ornstein-Uhlenbeck noise [38], particles that are subject to such an active noise exhibit non-Gaussian distributions under harmonic confinement, as demonstrated numerically in Ref. [39]. A characteristic trajectory of a Brownian particle in an active bath $[V(x) \equiv 0]$ is shown by the red line in Fig. 2(a). The mean square displacement (MSD) of this particle is shown by the symbols in Fig. 2(b). This MSD features a transition from a superdiffusive regime at short time scales to enhanced diffusion at long time scales. Fitting this MSD to its theoretical formula [40], i.e.,

$$
\operatorname{MSD}(t)=2 D_{\mathrm{p}} t+\frac{L_{\mathrm{a}}^{2}}{\tau_{\mathrm{a}}}\left[t-\tau_{\mathrm{a}}\left(1-e^{-t / \tau_{\mathrm{a}}}\right)\right],
$$

permits us to obtain the values of $\tau_{\mathrm{a}}=140 \mathrm{~ms}$ and $L_{\mathrm{a}}=$ $296 \mathrm{~nm}$. Importantly, the presence of the active noise introduces a memory in the fluctuation forces that is not matched by a corresponding memory in the friction term; this is a signature that the fluctuations of the probe particle are out of equilibrium.

We will now add the optical trap. In a weak trap ( $k=0.42 \mathrm{fN} \mathrm{nm}^{-1}$ and $\tau_{\mathrm{ot}}=190 \mathrm{~ms}$ ), the particle position distribution remains Gaussian, as shown by the experimental data (red symbols) in Fig. 1(d). Since the persistence length associated with the active bath $\left[L_{\mathrm{a}}\right.$, red bar in Fig. $\left.1(\mathrm{~d})\right]$ is much shorter than the characteristic dimension of the trap, the motion of the particle can be described as a standard Brownian motion in a harmonic potential, but at a higher effective temperature $T_{\text {eff }}=2300 \mathrm{~K}$, in agreement with previous works [40]. Thus, these data can be fitted to a Gaussian distribution (dashed red line), albeit with a variance larger than in the case of the thermal bath [Fig. 1(a)], despite the trap stiffness being the same:

$$
p_{\text {ot, eff }}(x)=Z_{\mathrm{ot}, \mathrm{eff}}^{-1} \exp \left(-\frac{k x^{2}}{2 k_{\mathrm{B}} T_{\mathrm{eff}}}\right),
$$

where $Z_{\mathrm{ot} \text {,eff }}=\sqrt{\frac{2 \pi k_{\mathrm{B}} T_{\mathrm{eff}}}{k}}$ is the normalization prefactor. 
However, as the trap stiffness increases, $L_{\mathrm{a}}$ becomes comparable to the characteristic dimension of the trap and the experimental position probability distribution [red symbols in Figs. 1(e) and 1(f)] become non-Gaussian, as can be seen by comparing these data with the best Gaussian fits (red dashed lines). This becomes particularly evident when considering the tails of the distribution, which are clearly heavier than expected from the Gaussian distributions. Importantly, these distributions are non-Boltzmann, which implies that the case of a particle in an active bath is qualitatively different from the case of a particle in a thermal bath. In fact, it is not possible to recover this probability distribution by altering the effective temperature of the system, as the position probability distribution associated with a Brownian particle in a harmonic potential is Gaussian at all temperatures. The alteration of the distribution is in fact due to the correlations introduced in the motion of the particle by the active bath, which lead to a non-Gaussian noise. Even though some works have demonstrated the possibility of having a Boltzmann distribution at long length scales [40] and others have implicitly indicated the breakdown of Boltzmann statistics at short length scales $[34,35,41]$, here we explicitly report a transition from a Boltzmann regime where it is possible to adopt a description based on effective temperatures to a non-Boltzmann regime where the system's deviation from a Boltzmann description is quantified.

The experimental probability distributions can be fitted using $q$-Gaussian distributions $[42,43]$, which are generalizations of the Gaussian distribution that depend on a parameter $q$ :

$$
p_{q}(x)=Z_{q}^{-1} \exp _{q}\left(-\frac{k x^{2}}{2 k_{\mathrm{B}} T_{\mathrm{eff}}}\right),
$$

where $Z_{q}$ is a normalization prefactor and $\exp _{q}(y)=[1+$ $(1-q) y]^{1 /(1-q)}$. Importantly, $q$-Gaussian distributions are characterized by heavy tails for $1<q<3$ and converge to the Gaussian distribution for $q \rightarrow 1$. The use of a $q$-Gaussian distribution permits us to quantify the deviation from a Gaussian distribution by using the fitting parameter $q$ : The greater the deviation of $q$ from 1 , the heavier the tail of the distribution. While the position probability distribution of the optically trapped particle in the thermal bath is well fitted by a Gaussian distribution at all stiffnesses $[q=0.997,1.000$, and 1.001 for Figs. 1(a), 1(b), and 1(c)], those in the active bath become more and more heavy tailed as the trap stiffness increases, i.e., $q=1.013,1.023$, and 1.142 for Figs. 1(d), 1(e), and $1(\mathrm{f})$, respectively.

We underline that this transition from a situation that can be described with Boltzmann statistics, albeit at a higher effective temperature, and a situation that cannot be described by Boltzmann statistics associated with the ratio between the characteristic scales of the trapping potential and the correlated active noise underlies several results connected with broken symmetries in active matter [34,35,41,44-47].

\section{NONEQUILIBRIUM RELATIONS AND FLUCTUATION THEOREMS IN ACTIVE BATHS}

Building on the results obtained in the previous section, we will now show that nonequilibrium relations cannot be applied in active baths according to their classical formulation. Furthermore, we will show that they can be recovered by introducing an effective potential. We will in focus particular on the Crooks fluctuation theorem [14], the Jarzynski equality [11], and the integral fluctuation theorem [12].

Let us consider a system driven from an initial to a final state such that the equilibrium free-energy difference between the two states is $\Delta F$ and the applied work is $W$. The Crooks fluctuation theorem [14] relates the probabilities of applying opposite works under forward and backward (time-reversed) protocols when a system is driven arbitrarily out of equilibrium [14]:

$$
\frac{P_{\mathrm{F}}(+\beta W)}{P_{\mathrm{R}}(-\beta W)}=e^{\beta(W-\Delta F)},
$$

where $\beta=\left(k_{\mathrm{B}} T\right)^{-1}, P_{\mathrm{F}}(+\beta W)$ is the probability of applying $+W$ work in a forward protocol, and $P_{\mathrm{R}}(-\beta W)$ is the probability of applying $-W$ work in the reversed protocol. An important precondition for the Crooks fluctuation theorem to be valid is that the system should initially be in a thermal equilibrium state and therefore have a Boltzmann distribution; since this initial condition is violated in an active bath [see Figs. 1(e) and 1(f)], we do not expect this relation to be verified in an active bath. The Jarzynski equality [11] allows one to obtain the free-energy difference from an exponential average of the work associated with irreversible realizations of a thermodynamic process:

$$
\left\langle e^{-\beta W_{i}}\right\rangle=e^{-\beta \Delta F},
$$

where $W_{i}$ is the $i$ th realization of the same protocol and angular brackets indicate an average over many realizations. Importantly, the initial (Boltzmann) equilibrium condition is needed also for the Jarzynski equality. Finally, a generalization of these results is the integral fluctuation theorem [12]

$$
\left\langle\exp \left(\beta Q-\ln \frac{p\left(x_{0}\right)}{\tilde{p}\left(\tilde{x}_{0}\right)}\right)\right\rangle=1,
$$

where $Q$ is the heat exchanged by the driven system with the surrounding environment and $p\left(x_{0}\right)$ and $\tilde{p}\left(\tilde{x}_{0}\right)$ are the initial and final probability distributions calculated at initial and final external parameters, respectively.

Experimentally, we consider a Brownian microparticle (silica, diameter $2 R=4.23 \pm 0.20 \mu \mathrm{m}$ ) held by a harmonic trap generated by focusing a laser beam (wavelength $\lambda=532 \mathrm{~nm}$, power $2.1 \mathrm{~mW}$ ) with a high-numerical-aperture objective (oil immersion, $100 \times$, NA equal to 1.30) [31]. The optical trap position can be translated using a spatial light modulator. The particle position is monitored by digital video microscopy with a spatial resolution of $5 \mathrm{~nm}$ and a sampling frequency of 50 frames per second. The resulting trapping potential is

$$
V_{\mathrm{ot}}(x, t)=\frac{1}{2} k[x-\lambda(t)]^{2},
$$

where $x$ is the position of the particle, $t$ is time, $k=1.33 \pm$ $0.05 \mathrm{fN} \mathrm{nm}^{-1}$ is the trap stiffness, and $\lambda(t)$ denotes the center of the harmonic potential, which acts as our external control parameter. In our protocol, $\lambda(t)$ is a square-wave function with amplitude $A$ and period $2 \tau$. First, the potential is centered at position 0 [dashed red line in Fig. 3(a)] for a time interval $\tau$. Then it is shifted to position $A=90 \mathrm{~nm}$ [solid red line in 

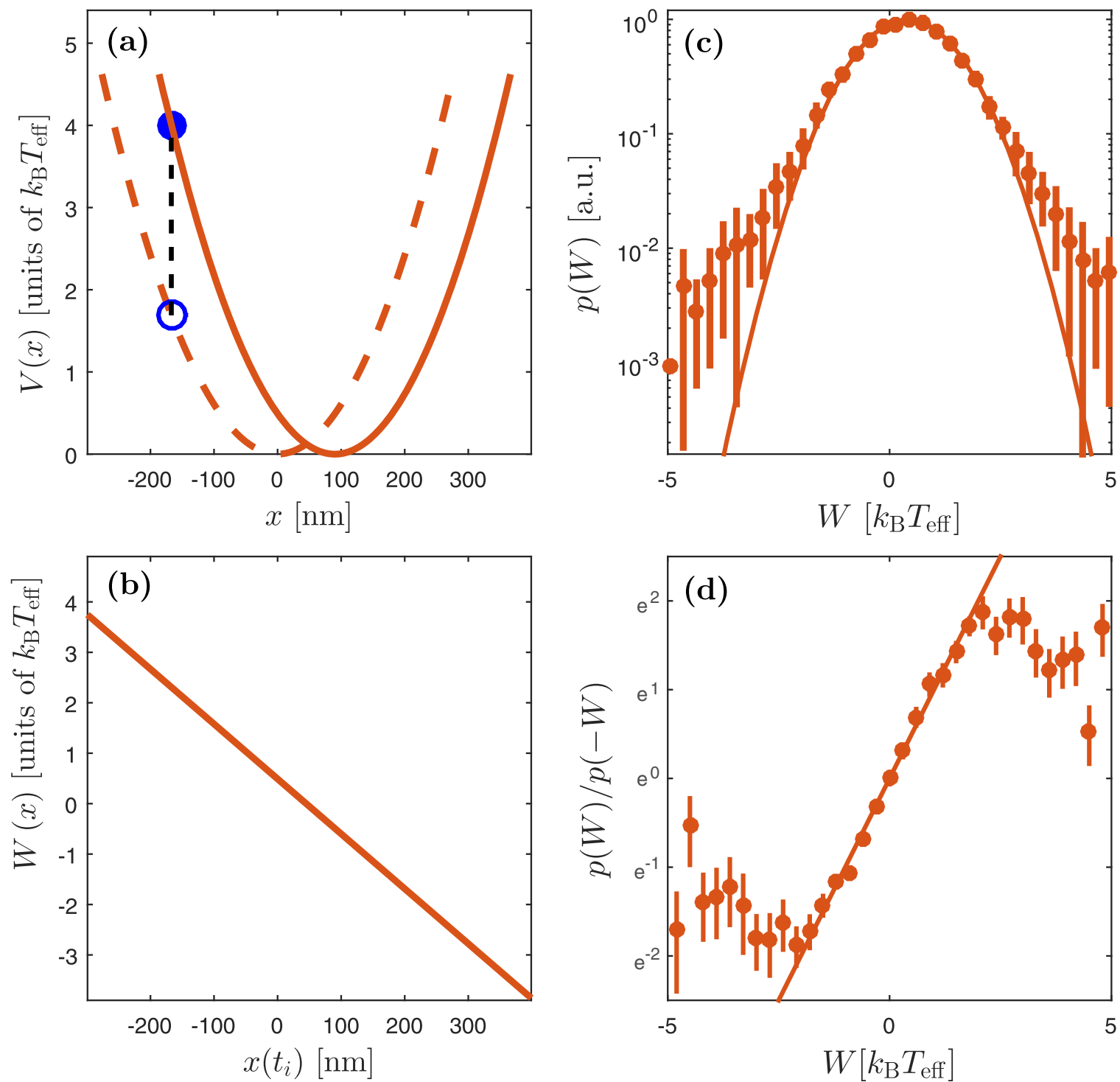

FIG. 3. Violation of the Crooks fluctuation theorem in an active bath. (a) The driving protocol entails an instantaneous translation of the trapping potential: The red dashed line represents the potential before switching, the red solid line the potential after switching, and the black dashed line the applied work. (b) Applied work as a function of the position of the particle before the potential translation. (c) Experimental probability distribution of work when the protocol shown in (a) is employed on a Brownian particle (silica, diameter $2 R=4.23 \pm 0.20 \mu \mathrm{m}$ ) held in an optical trap $\left(k=1.33 \mathrm{fN} \mathrm{nm}^{-1}\right)$ in an active (bacterial) bath, calculated using Eq. (16). The solid line represents the best Gaussian fit. (d) The red symbols show the ratio of the inverse work probabilities on the particle in active bath; they feature a clear deviation from the Crooks fluctuation theorem [solid line, Eq. (14)]. The data are obtained from 5000 work measurements; the error bars are obtained by repeating the measurements three times.

Fig. 3(a)], where it remains for an additional time interval $\tau$. Finally, the potential is brought back to position 0 and the protocol is iterated. The free energy of the optically trapped particle is

$$
F=-\frac{1}{\beta} \ln \left(Z_{\mathrm{ot}}\right),
$$

which depends only on $k$ and, importantly, not on the trap center position. Thus, since in our protocol the stiffness is kept constant, the free-energy difference between the initial and the final state is zero, i.e., $\Delta F=0$. Furthermore, because of symmetry, the forward and backward protocols are identical, i.e., $P_{\mathrm{F}}(y)=P_{\mathrm{R}}(y)$. Therefore, for our experimental realization, the Crooks fluctuation theorem (9) and Jarzynski equality (10) simplify to

$$
\frac{P(+W)}{P(-W)}=e^{\beta W}
$$

and

$$
\left\langle e^{-\beta W_{i}}\right\rangle=1 .
$$

We repeated 5000 times the experimental protocol described above and recorded the particle trajectory. Let $t_{i}$ be the time when the $i$ th potential switch occurs and the work $W_{i}$ applied on a particle during a sudden change of the external potential is equal to the instantaneous change in potential energy [dashed 

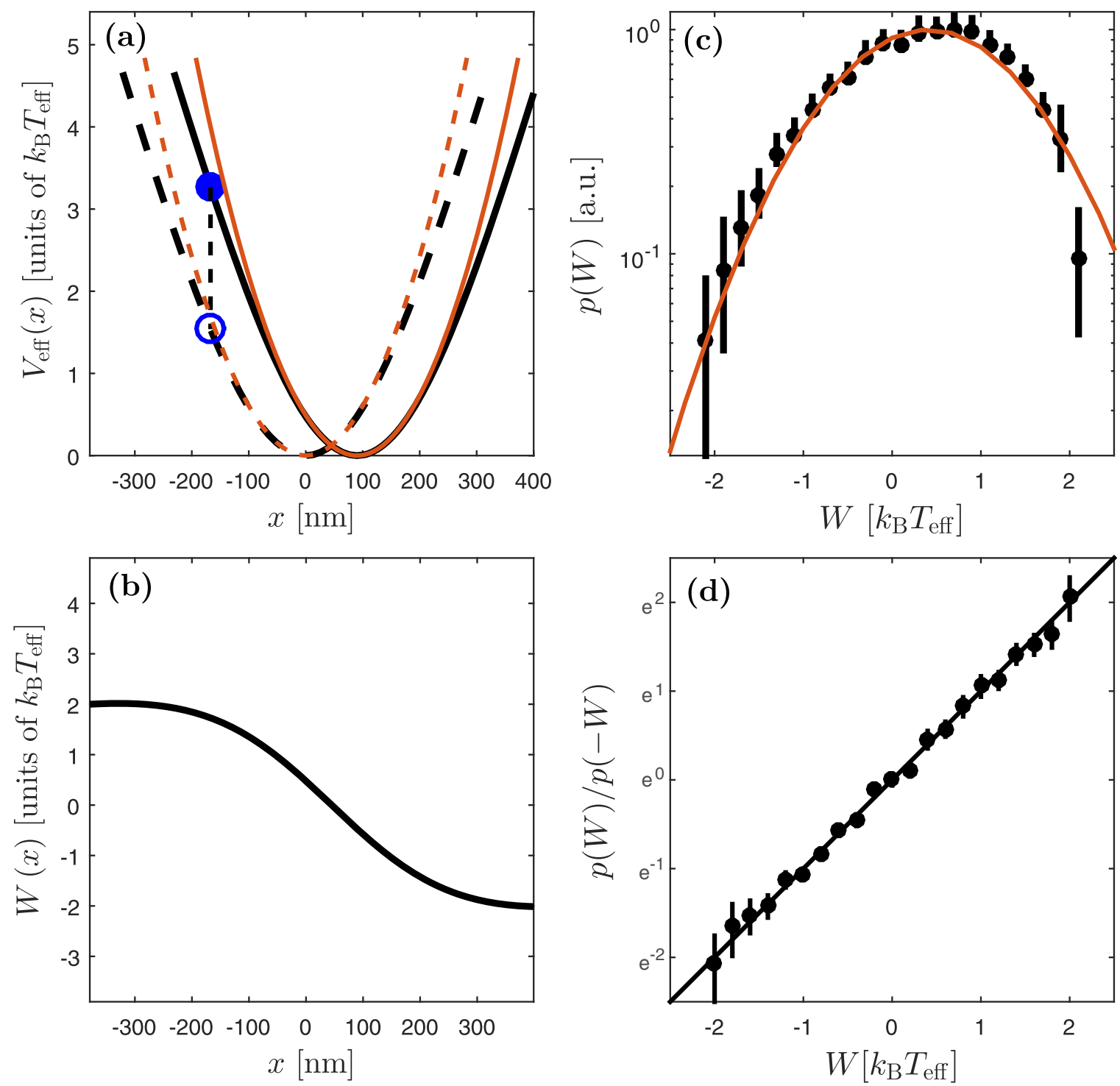

FIG. 4. Recovery of the Crooks fluctuation theorem in an active bath using effective potentials. (a) The driving protocol is the same as in Fig. 3(a), but effective potentials obtained from the particle's stationary position distribution (black lines) are employed instead of the optical potentials (red lines). The difference between the effective and the optical potentials is particularly evident in the tails. (b) Applied work as a function of the position of the particle before the potential translation. (c) Experimental probability distribution of work when the protocol shown in (a) is employed on a Brownian particle (silica, diameter $2 R=4.23 \pm 0.20 \mu \mathrm{m}$ ) held in an optical trap $\left(k=1.33 \mathrm{fN} \mathrm{nm}^{-1}\right)$ in an active (bacterial) bath, calculated with the effective potential approach [Eq. (19)]. The solid line is the same Gaussian fit as in Fig. 3(c). (d) The black symbols show the ratio of the inverse work probabilities on the particle in active bath: The use of effective potentials recovers the Crooks fluctuation theorem [solid line, Eq. (14)]. The data are obtained from 5000 work measurements; the error bars are obtained by repeating the measurements three times.

black line in Fig. 3(a)]; therefore,

$$
W_{i}=V_{\mathrm{ot}}\left(x\left(t_{i}\right), t_{i}^{+}\right)-V_{\mathrm{ot}}\left(x\left(t_{i}\right), t_{i}^{-}\right),
$$

where $V_{\mathrm{ot}}\left(x, t_{i}^{+}\right)$and $V_{\mathrm{ot}}\left(x, t_{i}^{-}\right)$are the potentials after and before the shift of the center, respectively. This quantity is shown in Fig. 3(b) as a function of the position of the particle at the switching time.

Initially, we performed our experiments in a thermal bath. Using the experimental work distributions obtained by Eq. (16), we verified that the Crooks fluctuation theorem (14) and Jarzynski equality (15) are satisfied in a thermal bath. In order to verify the integral fluctuation theorem (11), we need to evaluate the heat $Q$. According to the standard definition of heat in stochastic thermodynamics [48], the infinitesimal amount of heat exchanged along a stochastic trajectory reads $d Q=\partial_{x} V d x$. Thus, we measure the heat exchanged between the particle and the thermal bath during the interval $\left[t_{i}-\delta, t_{i}+\delta\right]$, which reads

$$
Q_{i}=V_{\mathrm{ot}}\left(x\left(t_{i}+\delta\right), t_{i}^{-}\right)-V_{\mathrm{ot}}\left(x\left(t_{i}-\delta\right), t_{i}^{-}\right),
$$

where $\delta=20 \mathrm{~ms}$ is significantly smaller than particle's relaxation time in the trap $\gamma / k(60 \mathrm{~ms})$ and thus the heat values are before relaxation. 
We then proceeded to test the nonequilibrium relations as given in Eqs. (14), (15), and (11) in an active bath employing the same protocol. We realize the active bath by using a bacterial bath, as in the experiments presented in the previous section.

In our first attempt, we use the optical potential $V_{\text {ot }}$ and introduce an effective temperature to account for the additional fluctuations introduced by the active bath. Doing so, we obtain an effective temperature $T_{\text {eff }}=810 \mathrm{~K}$ and consequently an effective energy scale $\beta_{\text {eff }}=\left(k_{\mathrm{B}} T_{\text {eff }}\right)^{-1}$, which we employ in Eq. (14). Importantly, the formulas to calculate the work applied on a particle [Eq. (16)] and heat exchange [Eq. (17)] are the same as in the case of the thermal bath described above. The resulting work distribution is represented by the symbols in Fig. 3(c). This distribution is not Gaussian, differently from the work distribution in the case of a thermal bath. In order to validate the Crooks fluctuation theorem (14), we estimate $P(+W) / P(-W)$, shown by the symbols in Fig. 3(d). We observe a clear deviation (particularly towards the tails) from the expected value [solid line in Fig. 3(d)], which shows that the Crooks fluctuation theorem is violated. We therefore conclude that the introduction of an effective temperature is not sufficient to employ nonequilibrium relations in an active bath. We remark that, even though the breakdown of the Crooks fluctuation theorem reported in Fig. 3(d) can be theoretically predicted from the fact that the particle does not start from an equilibrium ensemble, one of the central results of the current work is to show experimentally that this breakdown occurs for accessible parameters and can be quantified.

Therefore, in order to introduce a version of nonequilibrium relations working in active baths, we proceed to introduce an effective potential measured from the stationary particle distribution

$$
V_{\mathrm{eff}}(x, \lambda)=-k_{\mathrm{B}} T \ln \left(\frac{p^{\mathrm{s}}(x, \lambda)}{p^{\mathrm{s}}(0, \lambda)}\right),
$$

where $p^{\mathrm{s}}(x, \lambda)$ denotes the particle's stationary probability distribution under constant control parameter $\lambda$. An analogous approach was originally proposed by Hatano and Sasa for systems in nonequilibrium steady states in passive baths [49]: the authors introduced an effective potential to quantify the irreversibility of a process. The corresponding fluctuation theorem was experimentally verified for the case of a dragged Brownian particle, cycling between different steady states [50]. We experimentally measured $V_{\text {eff }}$ [Fig. 4(a)] by fitting the stationary distribution to a $q$-Gaussian function $(q=1.116)$. In this approach, Eq. (16) for the work applied on the particle when the potential is switched must be changed to

$$
W_{i}=V_{\mathrm{eff}}\left(x\left(t_{i}\right), t_{i}^{+}\right)-V_{\mathrm{eff}}\left(x\left(t_{i}\right), t_{i}^{-}\right) .
$$

The amount of work we apply during a switch as a function of the position of the particle at switching time is shown in Fig. 4(b) and the resulting work distribution is shown in Fig. 4(c); note the difference of the applied work using the effective potential [Fig. 4(b)] when compared with that using the optical potential [Fig. 3(b)], particularly in the tails of the distributions. Following from this work distribution we calculated $P(+W) / P(-W)$, which is shown by the symbols in Fig. 4(d). The effective potential approach enables us to recover the Crooks fluctuation theorem in an active bath, as can be seen from the fact the measured values [symbols in Fig. 4(d)] are in good agreement with the theoretical prediction [line in Fig. 4(d)].

To further corroborate the usefulness of the effective potential approach, we have also tested the Jarzynski equality (15) and the integral fluctuation theorem (11). Figure 5(a) shows that the Jarzynski equality is satisfied for large values of $\tau$ (we remind the reader that $\tau$ is the time interval between two successive switches of the potential), i.e., when the particle has enough time to reach a stationary state after switching the potential. Figure 5(b) shows that also the integral fluctuation theorem is validated when $\tau$ is large enough, as long as the formula for the heat [Eq. (17)] is calculated taking into account the effective potential, i.e.,

$$
Q_{i}=V_{\mathrm{eff}}\left(x\left(t_{i}+\delta\right), t_{i}^{-}\right)-V_{\mathrm{eff}}\left(x\left(t_{i}-\delta\right), t_{i}^{-}\right)
$$

It is important to underline that the recovery of the integral fluctuation theorem (11) is a more general result than the Crooks fluctuation theorem and Jarzynsky equality. Unlike the case of the Jarzynski equality (15) [Fig. 5(a)], which only requires the probability conservation for the sudden switch considered here, the fluctuations theorem for the total entropy (11) [Fig. 5(b)] is a sound result, which involves the entropy variation in the active bath $Q / T$, as given by Eq. (20), and the system entropy variation, as given by $-\ln \left[p^{\mathrm{s}}\left(x\left(t_{i}-\delta\right), t_{i}^{-}\right) / p^{\mathrm{s}}\left(x\left(t_{i}+\delta\right), t_{i}^{+}\right)\right]$, and thus generalizes the analogous result for the total entropy in passive baths [3].

\section{CONCLUSION}

We have shown that in an active bath, when a system is confined within a potential whose length scale is comparable to or smaller than the characteristic length scale associated with the active noise, non-Boltzmann statistics emerge. The introduction of an effective temperature is not sufficient to provide a framework within which to describe such systems. Such a framework can be instead provided by the use of an effective potential related to the system stationary distribution. This effective potential plays the same role as the mechanical potential; in particular, the stochastic work and heat can be obtained from it and such quantities turn out to satisfy the same fluctuation relations as the corresponding quantities in the passive bath case. We have exemplified these results with a series of experiments using an optically trapped particle in an active (bacterial) bath. Since active matter plays a central role in many systems, including very importantly living systems, our findings pose some significant limitations to the possibility of applying nonequilibrium fluctuation relations in their classical formulation to study this broad and valuable class of systems, pointing to the need for alternative approaches that explicitly model the presence of nonthermal fluctuations.

\section{ACKNOWLEDGMENTS}

A.-R.M. was partially supported by the Scientific and Technological Research Council of Turkey under Grant No. 114F207. A.I. was supported by the Danish Council for 

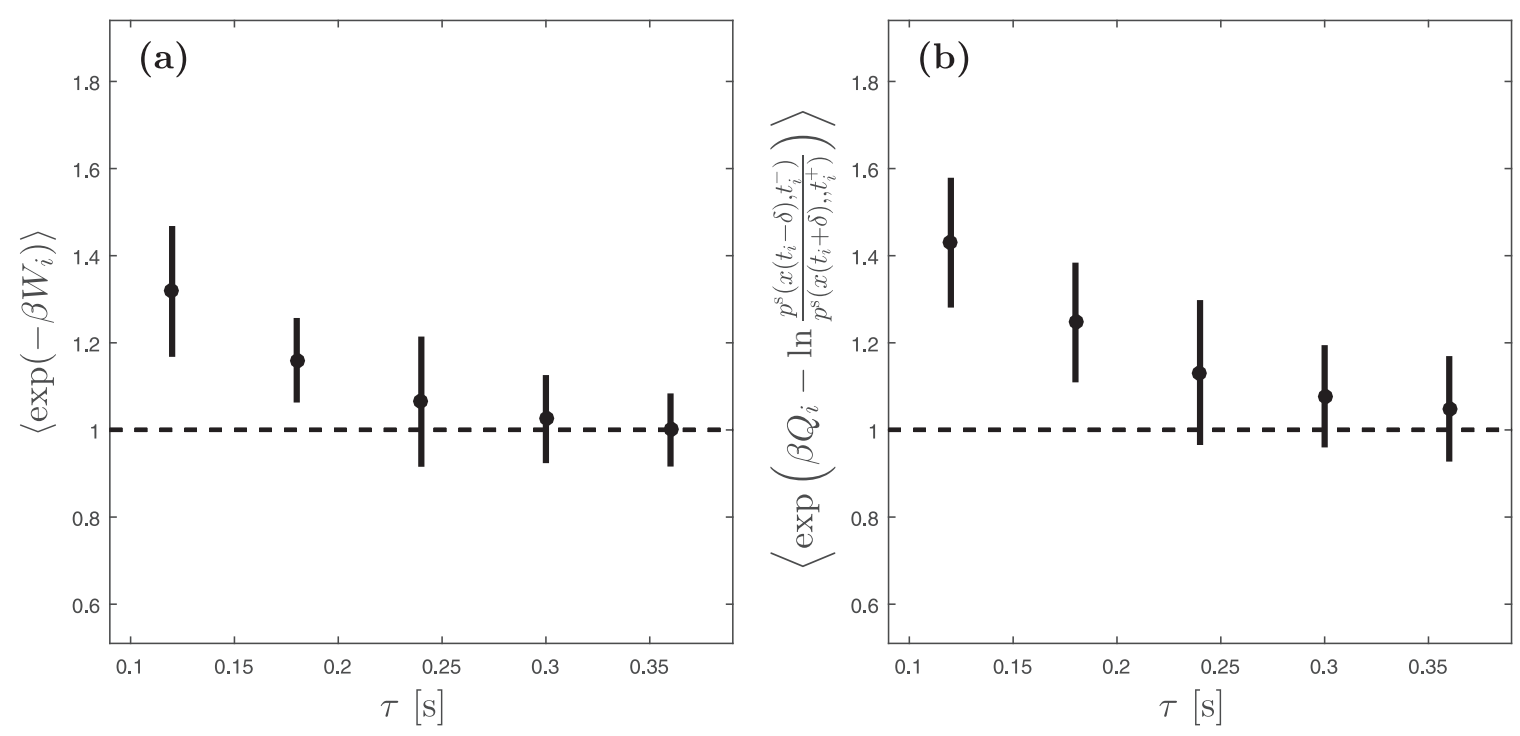

FIG. 5. Verification of the Jarzynski equality and integral fluctuation theorem in an active bath using effective potential. (a) Boltzmann weighted average of the applied work as a function of the waiting time $\tau$ between switches of the potential. If the particle is given enough time to reach a stationary state, the Jarzynski equality (15) is satisfied. Dots represent the average over all measured work values for each $\tau$. The rightmost dots $(\tau=360 \mathrm{~ms}$ ) correspond to the work distribution in Fig. 4(c). (b) Verification of the integral fluctuation theorem (11) using effective potentials. The heat $Q_{i}$ is calculated from each switch using Eq. (20). Note that the particle position probability $p^{\mathrm{s}}(x)$ is calculated before and after the switch, i.e., at $t_{i}^{-}$and $t_{i}^{+}$, respectively. The data are obtained from 5000 work measurements; the error bars are obtained by repeating the measurements three times.

Independent Research and the Villum Fonden. G.V. was partially supported by Marie Curie Career Integration Grant
No. PCIG11 GA-2012-321726 and a Distinguished Young Scientist award of the Turkish Academy of Sciences.
[1] J. W. Gibbs, Elementary Principles of Statistical Mechanics (Yale University Press, New Haven, 1902).

[2] C. Jarzynski, Equalities and inequalities: Irreversibility and the second law of thermodynamics at the nanoscale, Annu. Rev. Condens. Matter Phys. 2, 329 (2011).

[3] U. Seifert, Stochastic thermodynamics, fluctuation theorems, and molecular machines, Rep. Prog. Phys. 75, 126001 (2012).

[4] M. E. Cates, Diffusive transport without detailed balance in motile bacteria: Does microbiology need statistical physics, Rep. Prog. Phys. 75, 042601 (2012).

[5] D. J. Evans, E. G. D. Cohen, and G. P. Morriss, Probability of Second Law Violations in Nonequilibrium Steady States, Phys. Rev. Lett. 71, 2401 (1993).

[6] G. M. Wang, E. M. Sevick, E. Mittag, D. J. Searles, and D. J. Evans, Experimental Demonstration of Violations of the Second Law of Thermodynamics for Small Systems and Short Time Scales, Phys. Rev. Lett. 89, 050601 (2002).

[7] E. Barkai, Y. Garini, and R. Metzler, Strange kinetics of single molecules in living cells, Phys. Today 65(8), 29 (2012).

[8] C. Bechinger, R. Di Leonardo, H. Löwen, C. Reichhardt, G. Volpe, and G. Volpe, Active particles in complex and crowded environments, Rev. Mod. Phys. 88, 045006 (2016).

[9] S. Ciliberto, A. Imparato, A. Naert, and M. Tanase, Heat flux and entropy produced by thermal fluctuations, Phys. Rev. Lett. 110, 180601 (2013).

[10] A. Bérut, A. Imparato, A. Petrosyan, and S. Ciliberto, Stationary and Transient Fluctuation Theorems for Effective Heat Fluxes
Between Hydrodynamically Coupled Particles in Optical Traps, Phys. Rev. Lett. 116, 068301 (2016).

[11] C. Jarzynski, Nonequilibrium Equality for Free Energy Differences, Phys. Rev. Lett. 78, 2690 (1997).

[12] T. Speck and U. Seifert, Integral fluctuation theorem for the housekeeping heat, J. Phys. A: Math. Gen. 38, L581 (2005).

[13] G. Crooks, Nonequilibrium measurement of free energy differences for microscopically reversible Markovian systems, J. Stat. Phys. 90, 1481 (1998).

[14] G. E. Crooks, Entropy production fluctuation theorem and the nonequilibrium work relation for free energy differences, Phys. Rev. E 60, 2721 (1999).

[15] J. Liphardt, S. Dumont, S. B. Smith, I. Tinoco, Jr., and C. Bustamante, Equilibrium information from nonequilibrium measurements in an experimental test of Jarzynski's equality, Science 296, 1832 (2002).

[16] D. Collin, F. Ritort, C. Jarzynski, S. B. Smith, I. Tinoco, and C. Bustamante, Verification of the Crooks fluctuation theorem and recovery of RNA folding free energies, Nature (London) 437, 231 (2005).

[17] A. N. Gupta, A. Vincent, K. Neupane, H. Yu, F. Wang, and M. T. Woodside, Experimental validation of free-energylandscape reconstruction from non-equilibrium single-molecule force spectroscopy measurements, Nat. Phys. 7, 631 (2011).

[18] N. C. Harris, Y. Song, and C.-H. Kiang, Experimental Free Energy Surface Reconstruction from Single-Molecule Force Spectroscopy Using Jarzynski's Equality, Phys. Rev. Lett. 99, 068101 (2007). 
[19] V. Blickle, T. Speck, L. Helden, U. Seifert, and C. Bechinger, Thermodynamics of a Colloidal Particle in a Time-Dependent Nonharmonic Potential, Phys. Rev. Lett. 96, 070603 (2006).

[20] A. Imparato, F. Sbrana, and M. Vassalli, Reconstructing the free-energy landscape of a polyprotein by single-molecule experiments, Europhys. Lett. 82, 58006 (2008).

[21] S. An, J. Zhang, M. Um, D. Lv, Y. Lu, J. Zhang, Z. Yin, H. T. Quan, and K. Kim, Experimental test of the quantum Jarzynski equality with a trapped-ion system, Nat. Phys. 11, 193 (2015).

[22] R. Berkovich, J. Klafter, and M. Urbakh, Analyzing friction forces with the Jarzynski equality, J. Phys.: Condens. Matter 20, 354008 (2008).

[23] F. Douarche, S. Ciliberto, A. Petrosyan, and I. Rabbiosi, An experimental test of the Jarzynski equality in a mechanical experiment, Europhys. Lett. 70, 593 (2005).

[24] O.-P. Saira, Y. Yoon, T. Tanttu, M. Möttönen, D. V. Averin, and J. P. Pekola, Test of the Jarzynski and Crooks Fluctuation Relations in an Electronic System, Phys. Rev. Lett. 109, 180601 (2012).

[25] J. Harder, C. Valeriani, and A. Cacciuto, Activity-induced collapse and reexpansion of rigid polymers, Phys. Rev. E 90, 062312 (2014).

[26] R. Suzuki, C. A. Weber, E. Frey, and A. R. Bausch, Polar pattern formation in driven filament systems requires non-binary particle collisions, Nat. Phys. 11, 839 (2015).

[27] S. A. Mallory, C. Valeriani, and A. Cacciuto, Anomalous dynamics of an elastic membrane in an active fluid, Phys. Rev. E 92, 012314 (2015).

[28] J. Shin, A. G. Cherstvy, W. K. Kim, and R. Metzler, Facilitation of polymer looping and giant polymer diffusivity in crowded solutions of active particles, New J. Phys. 17, 113008 (2015).

[29] C. Battle, C. P. Broedersz, N. Fakhri, V. F. Geyer, J. Howard, C. F. Schmidt, and F. C. MacKintosh, Broken detailed balance at mesoscopic scales in active biological systems, Science 352, 604 (2016)

[30] H. Turlier, D. A. Fedosov, B. Audoly, T. Auth, N. S. Gov, C. Sykes, J.-F. Joanny, G. Gompper, and T. Betz, Equilibrium physics breakdown reveals the active nature of red blood cell flickering, Nat. Phys. 12, 513 (2016).

[31] P. H. Jones, O. M. Maragò, and G. Volpe, Optical Tweezers: Principles and Applications (Cambridge University Press, Cambridge, 2015).

[32] R. Parthasarathy, Rapid, accurate particle tracking by calculation of radial symmetry centers, Nat. Methods 9, 724 (2012).

[33] X.-L. Wu and A. Libchaber, Particle Diffusion in a Quasi-TwoDimensional Bacterial Bath, Phys. Rev. Lett. 84, 3017 (2000).

[34] E. Pinçe, S. K. P. Velu, A. Callegari, P. Elahi, S. Gigan, G. Volpe, and G. Volpe, Disorder-mediated crowd control in an active matter system, Nat. Commun. 7, 10907 (2016).
[35] N. Koumakis, C. Maggi, and R. Di Leonardo, Directed transport of active particles over asymmetric energy barriers, Soft Matter 10, 5695 (2014).

[36] D. T. N. Chen, A. W. C. Lau, L. A. Hough, M. F. Islam, M. Goulian, T. C. Lubensky, and A. G. Yodh, Fluctuations and Rheology in Active Bacterial Suspensions, Phys. Rev. Lett. 99, 148302 (2007).

[37] A. P. Solon, M. E. Cates, and J. Tailleur, Active Brownian particles and run-and-tumble particles: A comparative study, Eur. Phys. J. Spec. Top. 224, 1231 (2015).

[38] G. E. Uhlenbeck and L. S. Ornstein, On the theory of the Brownian motion, Phys. Rev. 36, 823 (1930).

[39] Z. Wang, H. Y. Chen, Y. J. Sheng, and H. K. Tsao, Diffusion, sedimentation equilibrium, and harmonic trapping of run-andtumble nanoswimmers, Soft Matter 10, 3209 (2014).

[40] C. Maggi, M. Paoluzzi, N. Pellicciotta, A. Lepore, L. Angelani, and R. Di Leonardo, Generalized Energy Equipartition in Harmonic Oscillators Driven by Active Baths, Phys. Rev. Lett. 113, 238303 (2014).

[41] N. Koumakis, A. Lepore, C. Maggi, and R. Di Leonardo, Targeted delivery of colloids by swimming bacteria, Nat. Commun. 4, 2588 (2013).

[42] C. Tsallis, Introduction to Nonextensive Statistical Mechanics (Springer, Heidelberg, 2009).

[43] G. B. Bagci and T. Oikonomou, Tsallis power laws and finite baths with negative heat capacity, Phys. Rev. E 88, 042126 (2013).

[44] A. Bricard, J. B. Caussin, D. Das, C. Savoie, V. Chikkadi, K. Shitara, O. Chepizhko, F. Peruani, D. Saintillan, and D. Bartolo, Emergent vortices in populations of colloidal rollers, Nat. Commun. 6, 7470 (2015).

[45] J. Tailleur and M. E. Cates, Sedimentation, trapping, and rectification of dilute bacteria, Europhys. Lett. 86, 60002 (2009).

[46] R. Di Leonardo, L. Angelani, D. DellArciprete, G. Ruocco, V. Iebba, S. Schippa, M. P. Conte, F. Mecarini, F. De Angelis, and E. Di Fabrizio, Bacterial ratchet motors, Proc. Natl. Acad. Sci. USA 107, 9541 (2010).

[47] L. Angelani and R. Di Leonardo, Geometrically biased random walks in bacteria-driven micro-shuttles, New J. Phys. 12, 113017 (2010).

[48] K. Sekimoto, Stochastic Energetics, Lecture Notes in Physics Vol. 799 (Springer, Heidelberg, 2010).

[49] T. Hatano and S.-i. Sasa, Steady-State Thermodynamics of Langevin Systems, Phys. Rev. Lett. 86, 3463 (2001).

[50] E. H. Trepagnier, C. Jarzynski, F. Ritort, G. E. Crooks, C. J. Bustamante, and J. Liphardt, Experimental test of Hatano and Sasa's nonequilibrium steady-state equality, Proc. Natl. Acad. Sci. USA 101, 15038 (2004). 